\title{
Impact of ${ }^{68}$ Ga-PSMA-11 PET/CT on the Management of Prostate Cancer Patients with Biochemical Recurrence
}

\author{
Jeremie Calais ${ }^{1}$, Wolfgang P. Fendler ${ }^{1}$, Matthias Eiber ${ }^{1}$, Jeannine Gartmann ${ }^{1}$, Fang-I Chu ${ }^{2}$, Nicholas G. Nickols ${ }^{2}$, \\ Robert E. Reiter ${ }^{3}$, Matthew B. Rettig ${ }^{3}$, Leonard S. Marks ${ }^{3}$, Thomas E. Ahlering ${ }^{4}$, Linda M. Huynh ${ }^{4}$, Roger Slavik ${ }^{1}$, \\ Pawan Gupta ${ }^{1}$, Andrew Quon ${ }^{1}$, Martin S. Allen-Auerbach ${ }^{1}$, Johannes Czernin ${ }^{1}$, and Ken Herrmann ${ }^{1}$ \\ ${ }^{I}$ Ahmanson Translational Imaging Division, Department of Molecular and Medical Pharmacology, UCLA Medical Center, Los \\ Angeles, California; ${ }^{2}$ Department of Radiation Oncology, UCLA Medical Center, Los Angeles, California; ${ }^{3}$ Department of Urology, \\ UCLA Medical Center, Los Angeles, California; and ${ }^{4}$ Department of Urology, UC Irvine Health, Irvine, California
}

See an invited perspective on this article on page 418 .

In this prospective survey of referring physicians, we investigated whether and how ${ }^{68} \mathrm{Ga}$-labeled prostate-specific membrane antigen 11 ( $\left.{ }^{68} \mathrm{Ga}-\mathrm{PSMA}-11\right) \mathrm{PET} / \mathrm{CT}$ affects the implemented management of prostate cancer patients with biochemical recurrence (BCR). Methods: We conducted a prospective survey of physicians (NCT02940262) who referred 161 patients with prostate cancer BCR (median prostatespecific antigen value, $1.7 \mathrm{ng} / \mathrm{mL}$; range, 0.05-202 ng/mL). Referring physicians completed one questionnaire before the scan to indicate the treatment plan without ${ }^{68} \mathrm{Ga}-\mathrm{PSMA}-11 \mathrm{PET} / \mathrm{CT}$ information (Q1; $n=101)$, one immediately after the scan to denote intended management changes (Q2; $n=101)$, and one 3-6 mo later to document the final implemented management (Q3; $n=56$ ). The implemented management was also obtained via electronic chart review or patient contact $(n=45)$. Results: A complete documented management strategy (Q1 + Q2 + implemented management) was available for 101 of 161 patients (63\%). Seventy-six of these (75\%) had a positive ${ }^{68} \mathrm{Ga}-\mathrm{PSMA}-11 \mathrm{PET} / \mathrm{CT}$ result. The implemented management differed from the prescan intended management (Q1) in 54 of 101 patients (53\%). The postscan intended management (Q2) differed from the prescan intended management (Q1) in 62 of 101 patients (61\%); however, these intended changes were not implemented in 29 of 62 patients (47\%). Pelvic nodal and extrapelvic metastatic disease on ${ }^{68} \mathrm{Ga}-\mathrm{PSMA}-11$ PET/CT (PSMA TON1M0 and PSMA TON1M1 patterns) was significantly associated with implemented management changes $(P=0.001$ and 0.05$)$. Conclusion: Information from ${ }^{68} \mathrm{Ga}-\mathrm{PSMA}-11 \mathrm{PET} / \mathrm{CT}$ brings about management changes in more than $50 \%$ of prostate cancer patients with BCR (54/101; 53\%). However, intended management changes early after ${ }^{68} \mathrm{Ga}-\mathrm{PSMA}-11 \mathrm{PET} / \mathrm{CT}$ frequently differ from implemented management changes.

Key Words: prostate cancer; biochemical recurrence; PET/CT; ${ }^{68} \mathrm{Ga}-\mathrm{PSMA}$; impact on implemented management

J Nucl Med 2018; 59:434-441

DOI: 10.2967/jnumed.117.202945

Received Oct. 3, 2017; revision accepted Nov. 30, 2017.

For correspondence or reprints contact: Jeremie Calais, David Geffen School of Medicine at UCLA, 10833 Le Conte Ave., Medical Plaza 200, Suite B114-61, Los Angeles, CA 90095.

E-mail: jcalais@mednet.ucla.edu

Guest Editor: Michael Graham, University of lowa

Published online Dec. 14, 2017.

COPYRIGHT (C 2018 by the Society of Nuclear Medicine and Molecular Imaging.
C ompared with conventional imaging, ${ }^{68} \mathrm{Ga}$-PSMA-11 PET/ $\mathrm{CT}$ is superior for detecting sites of prostate cancer biochemical recurrence (BCR) (1-4), is sensitive for detecting regional and distant metastatic disease $(3,5)$, is highly specific $(4$,$) and is asso-$ ciated with a low interreader variability (6).

Health-care providers and government agencies frequently judge the value of novel diagnostic tests by measuring their impact on patient management. Often, this impact has been estimated from survey information after the index test information becomes available to treating physicians (7). However, intended management early after imaging results become available does not necessarily translate into implemented management $(8-11)$. To our knowledge, the rate of implemented management changes related to ${ }^{68} \mathrm{Ga}-$ PSMA-11 PET/CT has not been determined prospectively in patients with BCR. Two retrospective studies attempted to determine rates of implemented management changes $(12,13)$, and 3 prospective studies evaluated the impact of ${ }^{68} \mathrm{Ga}$-PSMA-11 PET/CT on intended management changes $(1,14,15)$.

Here, we investigated prospectively the impact of ${ }^{68} \mathrm{Ga}-\mathrm{PSMA}-$ $11 \mathrm{PET} / \mathrm{CT}$ on the implemented management of prostate cancer patients with BCR.

\section{MATERIALS AND METHODS}

\section{Patients, Registration, and Authorization}

The Food and Drug Administration granted our investigational new drug application (NCT02940262) for a prospective study to evaluate the diagnostic performance of ${ }^{68} \mathrm{Ga}$-PSMA-11 PET/CT for localization of BCR. The primary endpoint of this study is the accuracy of ${ }^{68} \mathrm{Ga}-$ PSMA-11 PET/CT for identifying lesions. Here, we report on a secondary endpoint in a consecutively recruited subgroup of patients: the impact of ${ }^{68} \mathrm{Ga}$-PSMA-11 PET/CT on patient management. The University of California, Los Angeles (UCLA), Institutional Review Board approved the protocol, the informed consent forms, the participant information forms, and the prospective referring physician questionnaires (approval \#16-001095). From October 2016 to June 2017, we enrolled 161 patients with proven prostate adenocarcinoma and BCR after prostatectomy (prostate-specific antigen [PSA] level, $>0.2 \mathrm{ng} / \mathrm{mL}>6 \mathrm{wk}$ after surgery) or definitive radiotherapy (PSA rise, $\geq 2 \mathrm{ng} / \mathrm{mL}$ above the nadir). All patients provided written informed consent.

\section{Survey Design}

The survey design is depicted in Figure 1. We asked referring physicians to complete and return 3 questionnaires by email or fax. One questionnaire before the scan was required to indicate the treatment 


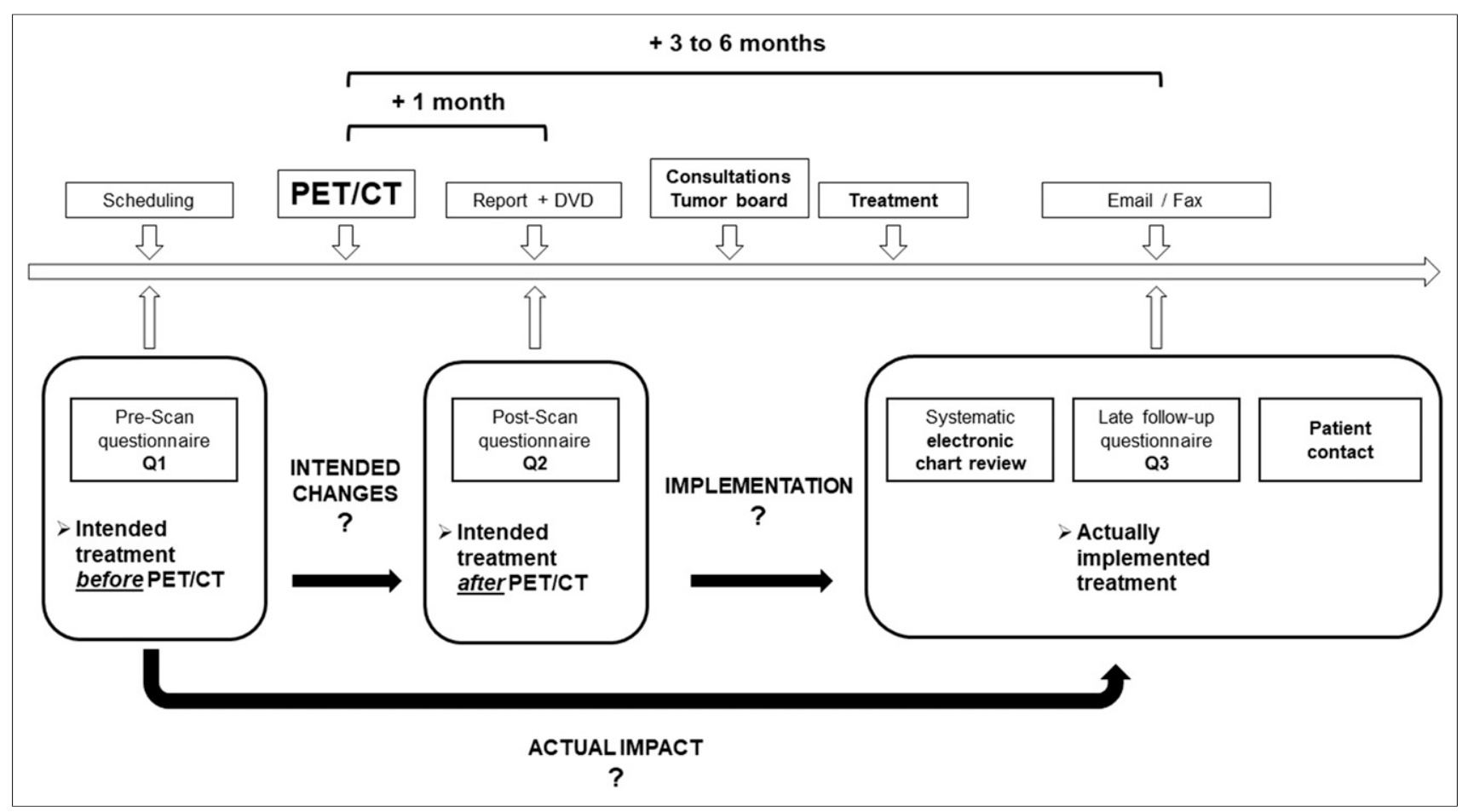

FIGURE 1. Study design.

plan without ${ }^{68}$ Ga-PSMA-11 PET/CT information (Q1). A second questionnaire inquired about intended management immediately after receipt of the written clinical report and the images (Q2). A final questionnaire emailed 3-6 mo later verified whether intended management changes were in fact implemented (Q3). Up to 3 email reminders were sent to referring physicians if questionnaires were not returned. To further document the implemented management strategy, we reviewed the electronic charts and followed up with patients.

The management options and changes are categorized in Table 1. We did not consider the addition or removal of androgen deprivation

TABLE 1

Treatment Options and Management Changes

\begin{tabular}{ll}
\hline \multicolumn{1}{c|}{ Parameter } & \multicolumn{1}{c}{ Description } \\
\hline Treatment option* & Salvage surgery \\
& Salvage radiation therapy \\
& Metastasis directed ablative radiation therapy (stereotactic body radiation therapy) \\
& Androgen deprivation therapy \\
& Chemotherapy \\
& Bone radionuclide therapy \\
& PSMA radionuclide therapy \\
& Other systemic treatment (vaccine therapy, immunotherapy) \\
& Active surveillance \\
& Conversion to focal treatment/new focal treatment (for either prostate bed, lymph node, \\
& or metastasis ablation) \\
& Conversion to systemic treatment \\
\hline Management change & Change in systemic treatment (adding new systemic treatment or removing systemic treatment) \\
& Conversion to active surveillance \\
\hline *Multiple treatment options were possible.
\end{tabular}


therapy to the treatment strategy to be a significant major management change except when active surveillance was intended or implemented.

Intended management changes between Q1 and Q2 represent the initial impact of imaging findings resulting in intended management changes, which may, however, not necessarily represent the implemented management change. Changes between Q2 and Q3/chart review/patient contact represent the difference between the intended postscan and implemented management plan. Changes between Q1 and $\mathrm{Q} 3 /$ chart review/patient contact represent the changes from prescan to the implemented management plan.

\section{${ }^{68}$ Ga-PSMA-11 PET/CT Protocol}

${ }^{68}$ Ga-PSMA-11 PET/CT imaging was performed according to recent guidelines (16) with a 64-detector PET/CT device (Biograph True Point 64 or Biograph mCT; Siemens). ${ }^{68}$ Ga-PSMA-11 (Glu-NH-CONH-Lys-(Ahx)- $\left.\left[{ }^{68} \mathrm{Ga}(\mathrm{HBED}-\mathrm{CC})\right]\right)$ was used as the PSMA ligand (17). The median injected dose was $196 \mathrm{MBq}$ (range, 93-241 $\mathrm{MBq}$ ). To reduce bladder activity, patients received $20 \mathrm{mg}$ of furosemide at the time of tracer injection if there was no contraindication. The median uptake period was $62 \mathrm{~min}$ (range, 52-96 min). A diagnostic CT scan (200-240 mAs, $120 \mathrm{kV}$ ) was performed after intravenous injection of contrast agent (if no contraindication), followed by the whole-body PET image acquisition (2-4 min/bed position). Standard image reconstruction parameters were used $(16,18)$.

\section{${ }^{68}$ Ga-PSMA-11 PET/CT Image Analysis}

${ }^{68} \mathrm{Ga}$-PSMA-11 PET/CT images were analyzed according to recent guidelines during clinical readouts by an experienced nuclear medicine physician who had unlimited access to all medical records $(16,18,19)$ : any focal uptake of ${ }^{68} \mathrm{Ga}$-PSMA-11 PET/CT above the background level and not associated with physiologic uptake or known pitfalls $(6,18)$ was considered PSMA-positive.

We routinely adopt an image-based TNM staging system and analyze the following regions for recurrence: prostate, prostate bed, and seminal vesicle remnants $(\mathrm{T})$, pelvic lymph nodes $(\mathrm{N})$ (internal iliac, obturator, external iliac, perirectal, presacral, common iliac, other), extrapelvic lymph nodes (M1a) (retroperitoneal, inguinal, chest, other), bone (M1b), and visceral organs (M1c).

\section{Statistics}

All variables were summarized by descriptive statistics (median and range). The comparisons for management change rates between
PSMA-positive and -negative patients were conducted using the $\chi^{2}$ test. Postscan intended management change (Q1 to Q2), nonimplementation of postscan intended management (Q2 to implemented), and implemented management change (Q1 to implemented) were considered 3 primary binary outcome variables in this study. Four potential predictor parameters were studied: National Comprehensive Cancer Network risk group, serum PSA level before ${ }^{68}$ Ga-PSMA-11 PET/CT, prior primary treatment (surgery or radiotherapy), and ${ }^{68} \mathrm{Ga}$-PSMA-11 PET/CT TNM pattern. Multiple logistic regression analysis was performed to investigate the potential association between these 4 predictors and the above 3 primary outcome variables. All statistical analyses were conducted in $\mathrm{R}(20)$.

\section{RESULTS}

\section{Referring Physicians and Questionnaires}

The flowchart is depicted in Figure 2. Fifty-seven physicians referred 161 patients for ${ }^{68} \mathrm{Ga}$-PSMA-11 PET/CT imaging. The complete documented management strategy $(\mathrm{Q} 1+\mathrm{Q} 2+$ actual implemented management) was available for 101 of 161 patients (63\%). Forty-two different physicians (10 from UCLA, 32 from other institutions) referred the 101 patients (38 from UCLA, 63 from other institutions).

Q1 and Q2 were completed in all 101 patients, and Q3 was completed in 56 of 101 patients (55\%). In 19 of these patients, electronic chart verification $(n=11)$ and patient contact $(n=$ 8) were used to further verify the accuracy of Q3 information. In the remaining 45 of 101 patients $(45 \%)$, Q3 was not completed, but electronic chart review $(n=32)$, patient contact $(n=10)$, or both $(n=3)$ was used to document the implemented management strategy (Fig. 2).

Referring physicians completed Q1 within a median of 18 $\mathrm{d}$ before the scan (range, 0-93 d). Q2 was completed within a median of $9 \mathrm{~d}$ after the scan (range, 1-89 d). We obtained information about the implemented treatment within medians of $105 \mathrm{~d}$ after the scan (range, 30-259 d) and $94 \mathrm{~d}$ after Q2 completion (range, 26-246 d).

\section{Patient Population}

Patient demographics are presented in Table 2. Briefly, in the 101 patients with a complete documented management strategy the median serum PSA value before ${ }^{68} \mathrm{Ga}$ PSMA-11 PET/CT was $1.7 \mathrm{ng} / \mathrm{mL}$ (range, 0.05-140 ng/mL). Eighty-seven of 101 patients $(86 \%)$ had prior prostatectomy, and 14 of $101(14 \%)$ had prior definitive radiotherapy. The median time between primary treatment and ${ }^{68} \mathrm{Ga}-\mathrm{PSMA}-11 \mathrm{PET} / \mathrm{CT}$ was $4.2 \mathrm{y}$ (range, $0.12-18 \mathrm{y}$ ), and 21 of 101 patients $(21 \%)$ had androgen deprivation therapy within 6 mo before ${ }^{68} \mathrm{Ga}$-PSMA-11 $\mathrm{PET} / \mathrm{CT}$.

In the 60 patients without a complete documented management strategy, the median serum PSA value before PET/CT was $2.25 \mathrm{ng} / \mathrm{mL}$ (range, 0.2-202 ng/mL).

\section{${ }^{68} \mathrm{Ga}-\mathrm{PSMA}-11$ PET/CT Findings}

${ }^{68} \mathrm{Ga}$-PSMA-11 PET/CT findings are detailed in Table 3. In brief, 76 of 101 patients (75\%) had a positive PET/CT study: 64

FIGURE 2. Flowchart. 
TABLE 2

Patient Characteristics $(n=101)$

\begin{tabular}{|c|c|}
\hline Parameter & Data \\
\hline \multicolumn{2}{|l|}{ Initial characteristics } \\
\hline Initial PSA at diagnosis, median $(\mathrm{ng} / \mathrm{mL})$ & $6.8(0.25-33.3)$ \\
\hline 10 & $52(52)$ \\
\hline$\geq 10$ to $<20$ & $10(10)$ \\
\hline$\geq 20$ & $9(9)$ \\
\hline Unknown & $30(30)$ \\
\hline \multicolumn{2}{|l|}{ Gleason score } \\
\hline$\leq 7$ & $32(32)$ \\
\hline$\geq 8$ & $66(66)$ \\
\hline Unknown & $3(3)$ \\
\hline \multicolumn{2}{|l|}{ Primary tumor stage } \\
\hline $\mathrm{T} 1-\mathrm{T} 2$ & $35(35)$ \\
\hline T3-T4 & $35(35)$ \\
\hline Unknown & $31(31)$ \\
\hline \multicolumn{2}{|l|}{ Initial NCCN risk group } \\
\hline Low & $5(5)$ \\
\hline Intermediate & $42(42)$ \\
\hline High & $44(44)$ \\
\hline N1 & $8(8)$ \\
\hline Unknown & $2(2)$ \\
\hline \multicolumn{2}{|l|}{ Prior treatment } \\
\hline Primary surgery & $87(86)$ \\
\hline Surgery only & $47(47)$ \\
\hline Surgery + ADT & $10(10)$ \\
\hline Surgery + SRT \pm ADT & $27(27)$ \\
\hline Surgery + SBRT $\pm A D T$ & $1(1)$ \\
\hline Surgery + chemotherapy \pm ADT & $2(2)$ \\
\hline Primary RT & $14(14)$ \\
\hline RT only & $5(5)$ \\
\hline $\mathrm{RT}+\mathrm{ADT}$ & $6(6)$ \\
\hline $\mathrm{RT}+\mathrm{SBRT} \pm \mathrm{ADT}$ & $2(2)$ \\
\hline $\mathrm{RT}+$ chemotherapy \pm ADT & $1(1)$ \\
\hline \multicolumn{2}{|l|}{$\mathrm{PET} / \mathrm{CT}$} \\
\hline Age at PET/CT, median (y) & $69(43-88)$ \\
\hline $\begin{array}{l}\text { Time between primary treatment and } \\
\text { PET/CT, median (y) }\end{array}$ & $4.2(0.12-18)$ \\
\hline ADT within 6 mo before imaging & $21(21)$ \\
\hline $\begin{array}{l}\text { Serum PSA before PET/CT, median } \\
(\mathrm{ng} / \mathrm{mL})\end{array}$ & $1.7(0.05-140)$ \\
\hline
\end{tabular}

NCCN $=$ National Comprehensive Cancer Network; ADT = androgen deprivation therapy; SRT = salvage radiation therapy; $\mathrm{RT}=$ radiation therapy; SBRT = stereotactic body radiation therapy.

Qualitative data are expressed as numbers followed by percentages in parentheses; continuous data are expressed as median followed by range in parentheses.

of $101(64 \%)$ had PSMA-positive intrapelvic lesions, and 37 of $101(37 \%)$ had PSMA-positive extrapelvic lesions. Thus far, histopathologic verification is available for 18 of the 76
PSMA-positive patients (24\%). PSMA-positive lesions corresponded to prostate adenocarcinoma in 14 of 18 patients $(78 \%)$ : in 4 of 7 (57\%) with local recurrence, in 7 of $7(100 \%)$ with pelvic LN recurrence, in 1 of 1 with retroperitoneal LN recurrence, and in 2 of $3(66 \%)$ with lung metastases reported by PET. The remaining 4 of 18 cases $(22 \%)$ may reflect ${ }^{68} \mathrm{Ga}$-PSMA-11 PET/CT falsepositive or biopsy false-negative findings.

Forty-seven of 60 patients $(78 \%)$ without a complete documented management strategy had a positive ${ }^{68} \mathrm{Ga}$-PSMA-11 PET/ CT study.

\section{Impact on Patient Management}

Management changes are detailed in Table 4. Implemented management changes (Q1 to implemented) were recorded for 54 of 101 patients (53\%). These consisted of conversion to focal treatment/new focal treatment in 29 of $101(29 \%)$, conversion to systemic treatment in 13 of 101 (13\%), change of systemic treatment approach in 5 of $101(5 \%)$, and conversion to active surveillance in 7 of $101(7 \%)$.

${ }^{68} \mathrm{Ga}-\mathrm{PSMA}-11 \mathrm{PET} / \mathrm{CT}$ (Q1 to Q2) resulted in intended management changes in 62 of 101 patients $(61 \%)$ : these included conversion to focal treatment/new focal treatment in 40 of 101 (40\%), conversion to systemic treatment in 12 of $101(12 \%)$, change in systemic treatment in 5 of $101(5 \%)$, and conversion to active surveillance in 5 of $101(5 \%)$.

Intended treatment as indicated in Q2 was implemented in 66 of 101 patients $(67.5 \%)$ : implementation of the intended strategy occurred in 33 of 62 patients with $(53 \%)$ and 33 of 39 patients without $(85 \%)$ intended management changes. Nonimplementation of intended management changes after the ${ }^{68}$ Ga-PSMA-11 PET/CT study (Q2) occurred in 35 of $101(35 \%)$ patients. Tumor board or other medical decisions $(13 / 35 ; 37 \%)$, patient choice $(11 / 35 ; 31 \%)$, and second opinions at other institutions $(5 / 35$; $14 \%$ ) accounted for nonimplementation. Reasons remained unknown

TABLE 3

${ }^{68} \mathrm{Ga}-\mathrm{PSMA}-11$ PET/CT Findings $(n=101)$

\begin{tabular}{|cc}
\hline \multicolumn{1}{c}{ Parameter } & Data \\
\hline${ }^{68}$ Ga-PSMA-11 PET/CT+ & $77(76)$ \\
\hline Prostate/prostate bed (T+) & $23(23)$ \\
\hline Pelvic LN (N1) & $47(47)$ \\
\hline Extrapelvic LN (M1a) & $21(21)$ \\
\hline Bone (M1b) & $19(19)$ \\
\hline Visceral (M1c) & $7(7)$ \\
\hline${ }^{68}$ Ga-PSMA-11 TNM pattern & \\
\hline PSMA T+ N0 M0 & $12(12)$ \\
\hline PSMA T0 N1 M0 & $25(25)$ \\
\hline PSMA T+ N1 M0 & $2(2)$ \\
\hline PSMA T+ N0 M1 & $6(6)$ \\
\hline PSMA T0 N0 M1 & $12(12)$ \\
\hline PSMA T0 N1 M1 & $17(17)$ \\
\hline PSMA T+ N1 M1 & $3(3)$ \\
\hline
\end{tabular}

$\mathrm{LN}=$ lymph node.

Data are numbers followed by percentages in parentheses. 
TABLE 4

Individual Management Changes

\begin{tabular}{|c|c|c|c|}
\hline Management change & Q1 to Q2 & Q2 to implemented & Q1 to implemented \\
\hline Conversion to focal treatment/new focal treatment & $40(40)$ & $14(14)$ & $29(29)$ \\
\hline Active surveillance to surgery $\pm A D T$ & $5(5)$ & $0(0)$ & $5(5)$ \\
\hline Active surveillance to $\mathrm{SRT} \pm \mathrm{ADT}$ & $9(9)$ & $2(2)$ & $3(3)$ \\
\hline Active surveillance to $\mathrm{SBRT} \pm \mathrm{ADT}$ & $0(0)$ & $0(0)$ & $1(1)$ \\
\hline$A D T$ to surgery $\pm A D T$ & $3(3)$ & $0(0)$ & $1(1)$ \\
\hline ADT to surgery + SRT + ADT & $0(0)$ & $0(0)$ & $1(1)$ \\
\hline ADT to $S R T \pm A D T$ & $4(4)$ & $1(1)$ & $2(2)$ \\
\hline ADT to SRT + CTx + ADT & $1(1)$ & $0(0)$ & $0(0)$ \\
\hline $\mathrm{ADT}$ to $\mathrm{SBRT} \pm \mathrm{ADT}$ & $4(4)$ & $0(0)$ & $4(4)$ \\
\hline $\mathrm{ADT}$ to $\mathrm{SBRT}+\mathrm{CTx}+\mathrm{ADT}$ & $0(0)$ & $0(0)$ & $1(1)$ \\
\hline CTx + ADT to surgery & $1(1)$ & $0(0)$ & $0(0)$ \\
\hline $\mathrm{CTx}+\mathrm{ADT}$ to $\mathrm{SRT} \pm \mathrm{ADT}$ & $1(1)$ & $1(1)$ & $1(1)$ \\
\hline $\mathrm{CTx}+\mathrm{ADT}$ to $\mathrm{SBRT} \pm \mathrm{ADT}$ & $1(1)$ & $0(0)$ & $2(2)$ \\
\hline PSMA-RNT to SRT + ADT & $0(0)$ & $1(1)$ & $0(0)$ \\
\hline SRT $\pm A D T$ to surgery & $5(5)$ & $2(2)$ & $2(2)$ \\
\hline $\mathrm{SRT} \pm \mathrm{ADT}$ to $\mathrm{SBRT} \pm \mathrm{ADT}$ & $5(5)$ & $1(1)$ & $5(5)$ \\
\hline $\mathrm{SRT} \pm \mathrm{ADT}$ to $\mathrm{SBRT}+\mathrm{SRT} \pm \mathrm{ADT}$ & $0(0)$ & $1(1)$ & $1(1)$ \\
\hline $\mathrm{SRT} \pm \mathrm{ADT}$ to $\mathrm{SBRT}+\mathrm{CT} x+\mathrm{ADT}$ & $0(0)$ & $1(1)$ & $0(0)$ \\
\hline $\mathrm{SRT}+\mathrm{CT} x+\mathrm{ADT}$ to SBRT + ADT & $0(0)$ & $1(1)$ & $0(0)$ \\
\hline SBRT + ADT to surgery & $1(1)$ & $0(0)$ & $0(0)$ \\
\hline Surgery + ADT to SRT + ADT & $0(0)$ & $2(2)$ & $0(0)$ \\
\hline Surgery + ADT to surgery + SRT + ADT & $0(0)$ & $1(1)$ & $0(0)$ \\
\hline Conversion to systemic treatment & $12(12)$ & $7(7)$ & $13(13)$ \\
\hline Active surveillance to ADT & $4(4)$ & $1(1)$ & $4(4)$ \\
\hline Active surveillance to other systemic treatment + ADT & $0(0)$ & $0(0)$ & $3(3)$ \\
\hline Surgery $\pm A D T$ to $A D T$ & $0(0)$ & $3(3)$ & $0(0)$ \\
\hline $\mathrm{SRT}+\mathrm{CTx} \pm \mathrm{ADT}$ to $\mathrm{ADT}$ & $1(1)$ & $0(0)$ & $1(1)$ \\
\hline $\mathrm{SRT} \pm \mathrm{ADT}$ to $\mathrm{ADT}$ & $5(5)$ & $2(2)$ & $5(5)$ \\
\hline $\mathrm{SRT} \pm \mathrm{ADT}$ to $\mathrm{CT} x+\mathrm{ADT}$ & $1(1)$ & $0(0)$ & $0(0)$ \\
\hline SBRT + ADT to other systemic treatment $+\mathrm{ADT}$ & $1(1)$ & $1(1)$ & $0(0)$ \\
\hline Unknown to ADT & $0(0)$ & $0(0)$ & $0(0)$ \\
\hline Conversion to active surveillance & $5(5)$ & $7(7)$ & $7(7)$ \\
\hline Surgery $\pm A D T$ to active surveillance & $0(0)$ & $3(3)$ & $0(0)$ \\
\hline $\mathrm{SRT} \pm \mathrm{ADT}$ to active surveillance & $4(4)$ & $2(2)$ & $4(4)$ \\
\hline SRT + CTx to active surveillance & $0(0)$ & $0(0)$ & $0(0)$ \\
\hline ADT to active surveillance & $1(1)$ & $2(2)$ & $3(3)$ \\
\hline Change in systemic treatment & $5(5)$ & $7(7)$ & $5(5)$ \\
\hline$A D T$ to $C T x \pm A D T$ & $3(3)$ & $0(0)$ & $0(0)$ \\
\hline ADT to other systemic treatment $\pm A D T$ & $0(0)$ & $4(4)$ & $2(2)$ \\
\hline$C T x \pm A D T$ to $A D T$ & $0(0)$ & $3(3)$ & $1(1)$ \\
\hline $\mathrm{CTx}+$ bone RNT + ADT to ADT + CTx & $0(0)$ & $0(0)$ & $0(0)$ \\
\hline CTx + bone RNT + ADT to CTx + PSMA-RNT + ADT & $0(0)$ & $0(0)$ & $0(0)$ \\
\hline CTx + bone RNT + ADT to PSMA-RNT & $1(1)$ & $0(0)$ & $1(1)$ \\
\hline Bone RNT + ADT to CTx + ADT & $1(1)$ & $0(0)$ & $1(1)$ \\
\hline Bone RNT + ADT to PSMA-RNT & $0(0)$ & $0(0)$ & $0(0)$ \\
\hline Total & $62(61)$ & $35(35)$ & $54(53)$ \\
\hline
\end{tabular}

ADT = androgen deprivation therapy; SRT = salvage radiation therapy; SBRT = stereotactic body radiation therapy; CTx = chemotherapy; RNT = radionuclide therapy.

Data are numbers followed by percentages in parentheses. 


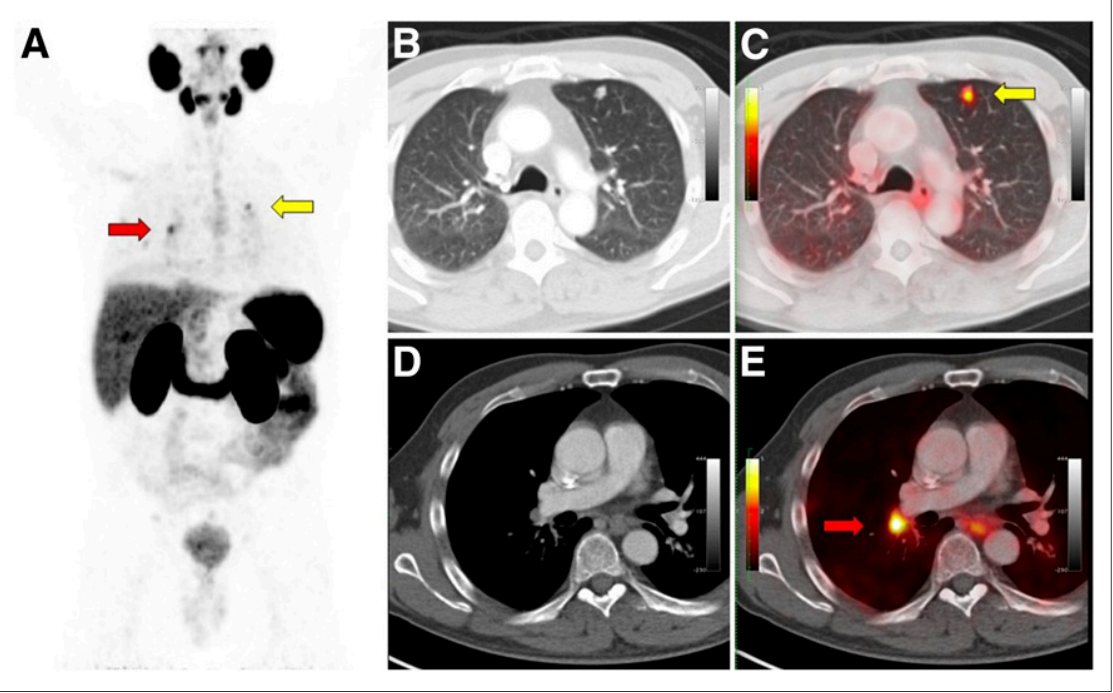

FIGURE 3. A $67-y$-old man with BCR (PSA level, $10.7 \mathrm{ng} / \mathrm{mL}$; doubling time, $9.3 \mathrm{mo}$ ) of initially high-risk prostate cancer (Gleason 9; pT3a) 8 y after primary radical prostatectomy and adjuvant prostate bed irradiation. Intended prescan treatment was androgen deprivation therapy. ${ }^{68} \mathrm{Ga}-\mathrm{PSMA}-11 \mathrm{PET} / \mathrm{CT}$ showed intense ${ }^{68} \mathrm{Ga}-\mathrm{PSMA}-11$ uptake (SUV $\left.\max , 7\right)$ in multiple lung nodules (yellow arrows) and thoracic lymph nodes (red arrows). Intended postscan treatment (Q2) was chemotherapy plus androgen deprivation therapy. CT-guided biopsy of upper left lung nodule confirmed metastatic prostatic adenocarcinoma. Patient elected to forgo chemotherapy because of potential side effects, and thus, intended postscan management (Q2) was not implemented. Actual management was androgen deprivation therapy alone, and thus, there was no change from prescan intended management as recorded on Q1. (A) ${ }^{68}$ Ga-PSMA-11 PET maximum-intensity projection. (B) Axial CT, lung window. (C) Axial ${ }^{68} \mathrm{Ga}-\mathrm{PSMA}-11 \mathrm{PET} / \mathrm{CT}$, lung window. (D) Axial CT, mediastinal window. (E) Axial ${ }^{68} \mathrm{Ga}-\mathrm{PSMA}-11 \mathrm{PET} / \mathrm{CT}$, mediastinal window.

in 6 of $35(17 \%)$. Figures 3 and 4 depict patients in whom subsequent decisions led to nonimplementation of intended management.

\section{Predictors of Management Changes}

Among 4 tested parameters (National Comprehensive Cancer Network risk group, PSA level before PET/CT, prior primary treatment, and ${ }^{68} \mathrm{Ga}$-PSMA-11 TNM pattern), the ${ }^{68} \mathrm{Ga}$-PSMA-11 PET/CT TNM pattern was the only significant predictor of intended (Q1 to Q2) and implemented (Q1 to implemented) management changes.

Specifically, the probability of having an intended management change was higher in patients with pelvic nodal disease only (PSMA TON1M0) than in patients with negative scans $(P=0.02)$. Furthermore, intended management changes occurred more frequently in patients with positive ${ }^{68} \mathrm{Ga}$-PSMA$11 \mathrm{PET} / \mathrm{CT}$ scans $(52 / 76 ; 68 \%)$ than in those with negative scans $(10 / 25 ; 40 \%)(P=0.02)$. Figure 5 illustrates a PSMA T0N1M0 pattern.

The probability of having implemented management changes (Q1 to implemented) was higher in patients with PSMA T0N1M0 $(P=$ $0.001)$ and T0N1M1 patterns $(P=0.05)$ than in those with negative scans. Finally, implemented management changes (Q1 to implemented) occurred more frequently in patients with positive ${ }^{68} \mathrm{Ga}-$ PSMA-11 PET/CT scans $(48 / 76 ; 63 \%)$ than in those with negative scans $(6 / 25 ; 24 \%)(P<0.002)$.

None of the 4 parameters predicted nonimplementation of the intended management after the ${ }^{68}$ Ga-PSMA-11 PET/CT study (Q2 to implemented).

\section{DISCUSSION}

This prospective survey enabled a systematic assessment of how referring physicians respond to the diagnostic information provided by ${ }^{68} \mathrm{Ga}-\mathrm{PSMA}-11 \mathrm{PET} / \mathrm{CT}$ imaging. The implemented management differed from the prescan treatment plan (Q1) in 54 of 101 patients (53\%). PSMA T0N1M0 and PSMA T0N1M1 patterns were significantly associated with a higher likelihood of implemented management changes $(P=0.001$ and 0.05 , respectively) (Fig. 5). These 2 patterns frequently lead to focal therapy with surgery or radiation therapy (especially pelvic node-only recurrence), a strategy that can be considered only after scan findings are available.

A significant impact of any diagnostic test on management suggests value for patients and is a prerequisite for widespread acceptance (7). However, one concern about studies using intended management changes as an endpoint is that these changes may not be implemented (8-11). The implemented management reflects the true impact of an index test $(8,9)$. In fact, implemented management changes are the most reliable source for cost or cost-effectiveness analyses $(8,21,22)$. Implemented management can be assessed retrospectively-for instance, from large databases (22). However, intended management before image information becomes available can be determined only prospectively. Thus, reliable information must arise from information that is prospectively recorded before and after the index test is performed.

We documented actual management changes in 53\% of prostate cancer patients with BCR in response to ${ }^{68} \mathrm{Ga}-\mathrm{PSMA}-11 \mathrm{PET} / \mathrm{CT}$ imaging. These findings are in line with the mean pooled rate of $57.3 \%$ (range, $39 \%-76 \%$ ) from retrospective studies $(12,13)$. The current rate of intended management changes in $61 \%$ of patients is also consistent with the pooled rate of $55.8 \%$ (range, 51\%-63\%) from prospective studies $(1,14,15)$. Other studies enrolled patients prospectively for other reasons, but management changes were not assessed prospectively (23).

We demonstrated that in 1 of 3 patients, the intended management changes (Q2) were not implemented, consistent with previous studies (8). Prostate cancer patients are offered multiple treatment options, including androgen deprivation therapy, surgery, radiotherapy, or combinations of these. Clinical decisions are often based on imaging information, tumor boards, expert opinions, and patient preference. The timing of surveys conducted early after the index test, in our case Q2, precludes consideration of other factors that can affect final decision making. Imaging is obviously not the only determinant of management decisions. These are often based on tumor board or expert opinions, second opinions, patient preference, and other factors. Thus, intended management changes, used as study endpoints in many studies, do not provide actual patient management information. In the current study, intended management changes were often either not implemented or changed to yet other management plans after more information became available to 
referring physicians. Interestingly, the rate of nonimplementation was much higher in patients with intended changes after the scan (47\%) than in patients without intended changes $(15 \%)$. The current findings underscore a severe limitation of surveys using intended management changes as an endpoint: surveys cannot detect changes induced by tumor board or expert opinions, patient preference, and other factors. To clearly define the implemented management, verification of the management plan using other sources such as electronic chart review, patient information, and clinician information is a prerequisite for appropriate assessment.

Complete information was available for only 101 of 161 patients $(63 \%)(24-26)$. This less than $100 \%$ completion rate may have introduced a responder bias. However, because 96 of 161 patients $(60 \%)$ were referred from different external institutions, we considered a $63 \%$ completion rate as satisfactory. In addition, the large number of participating physicians $(n>40)$ argues against a significant bias. Furthermore, imaging findings and clinical parameters were comparable between the 101 patients with a complete documented management strategy and the 60 patients without (detection rate of $75 \%$ vs. $78 \%$; median PSA levels of $1.7 \mathrm{ng} / \mathrm{mL}$ [range, $0.05-140 \mathrm{ng} / \mathrm{mL}$ ] vs. $2.25 \mathrm{ng} / \mathrm{mL}$ [range, $0.2-202 \mathrm{ng} / \mathrm{mL}$ ]). The relatively low completion rate of Q3 is a negligible problem, because we verified implemented management strategies via other means (electronic chart review and contact with patients).

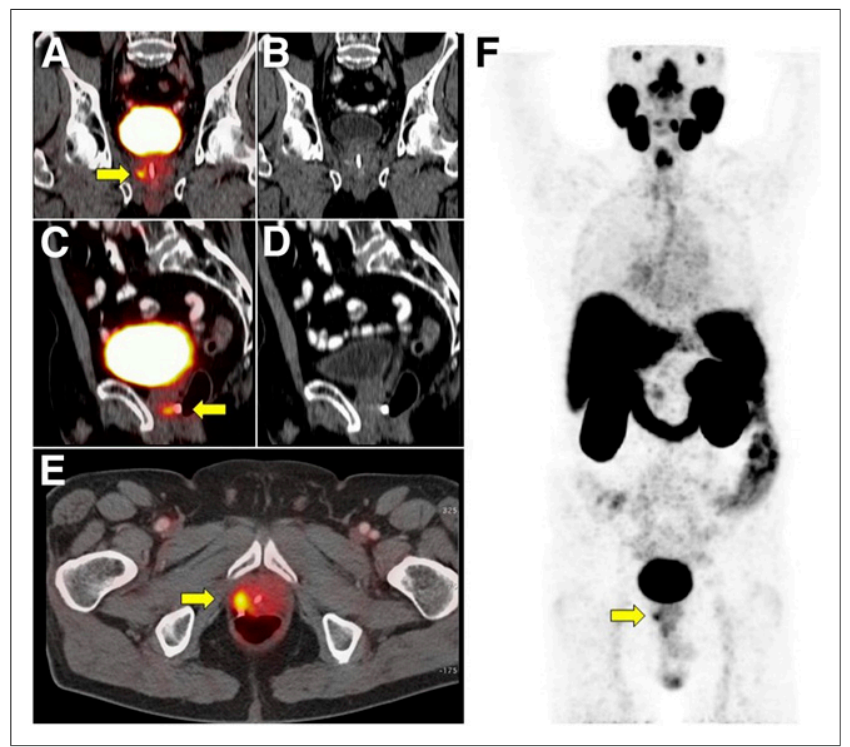

FIGURE 4. ${ }^{68} \mathrm{Ga}-\mathrm{PSMA}-11 \mathrm{PET} / \mathrm{CT}$ in $75-\mathrm{y}$-old man with BCR (PSA level, $2.88 \mathrm{ng} / \mathrm{mL}$; doubling time, $4.5 \mathrm{mo}$ ) of initially high-risk prostate cancer (Gleason score, 8; pT3) 4 y after primary radiotherapy without androgen deprivation therapy. Q1 listed androgen deprivation therapy as planned treatment. ${ }^{68} \mathrm{Ga}-\mathrm{PSMA}-11$ PET/CT showed focal ${ }^{68} \mathrm{Ga}-$ PSMA-11 uptake (SUV $\max , 3.9$ ) in right prostate lobe (yellow arrows). Intended treatment after ${ }^{68} \mathrm{Ga}-\mathrm{PSMA}-11$ PET/CT (Q2) was surgery, which patient refused because of potential side effects. Actual management was thus androgen deprivation therapy as indicated on Q1 (no management change). (A) Coronal ${ }^{68} \mathrm{Ga}-\mathrm{PSMA}-11 \mathrm{PET} / \mathrm{CT}$. (B) Coronal CT. (C) Sagittal ${ }^{68}$ Ga-PSMA-11 PET/CT. (D) Sagittal CT. (E) Axial ${ }^{68} \mathrm{Ga}-\mathrm{PSMA}-11$ PET/CT. (F) ${ }^{68} \mathrm{Ga}-\mathrm{PSMA}-11$ PET maximum-intensity projection. Yellow arrows denote focal tracer uptake consistent with intraprostatic recurrence.

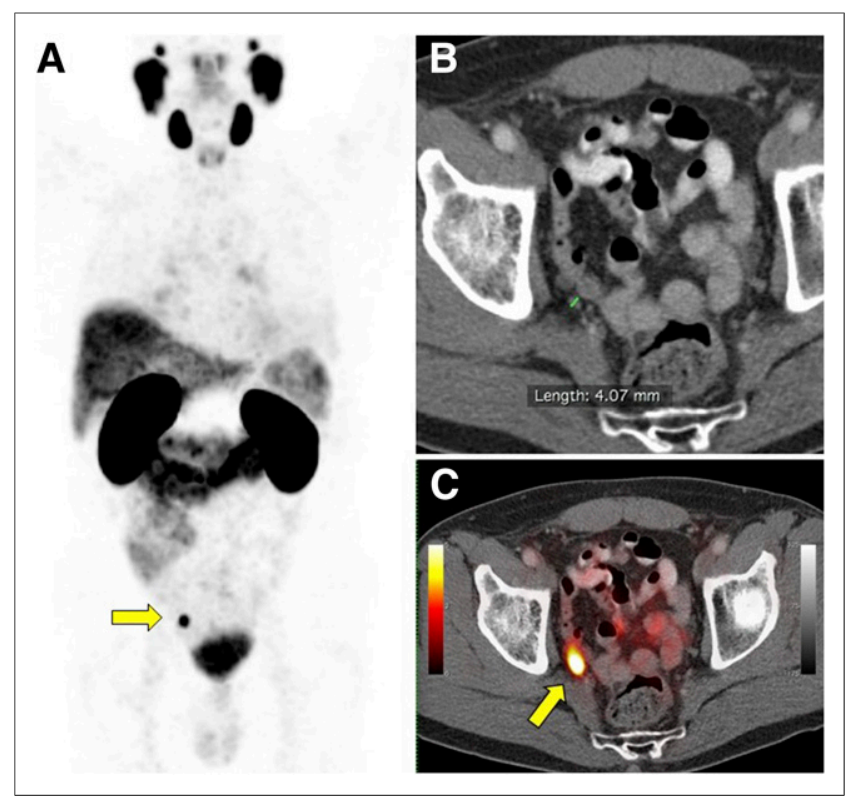

FIGURE 5. ${ }^{68} \mathrm{Ga}-\mathrm{PSMA}-11 \mathrm{PET} / \mathrm{CT}$ scan in 70-y-old man with BCR (PSA level, $1.06 \mathrm{ng} / \mathrm{mL}$; doubling time, $7 \mathrm{mo}$ ) of initially high-risk prostate cancer (Gleason score, 8; pT2c) 1 y after primary radical prostatectomy. Intended treatment before ${ }^{6} \mathrm{Ga}-\mathrm{PSMA}-11 \mathrm{PET} / \mathrm{CT}$ was active surveillance (Q1). ${ }^{68 \mathrm{Ga}-}$ PSMA-11 PET/CT showed intense ${ }^{68} \mathrm{Ga}-\mathrm{PSMA}-11$ uptake (SUV $\left.\max , 10.8\right)$ in 4-mm right internal iliac pelvic lymph node (yellow arrows). Intended treatment after ${ }^{68} \mathrm{Ga}-\mathrm{PSMA}-11$ PET/CT (Q2) was surgery, which was implemented. Patient underwent pelvic lymph node dissection 2 mo later, which confirmed metastatic prostatic adenocarcinoma. (A) ${ }^{68} \mathrm{Ga}-\mathrm{PSMA}-11$ PET maximum-intensity projection. (B) Axial CT. (C) Axial ${ }^{68} \mathrm{Ga}-\mathrm{PSMA}-11 \mathrm{PET} / \mathrm{CT}$.

\section{CONCLUSION}

This prospective referring physician-based survey shows a significant impact $(54 / 101 ; 53 \%)$ of ${ }^{68} \mathrm{Ga}$-PSMA-11 PET/CT on the actual management of prostate cancer patients with BCR. Importantly, intended management changes after ${ }^{68} \mathrm{Ga}-\mathrm{PSMA}-11 \mathrm{PET} / \mathrm{CT}$ were further modified in almost $50 \%$ of the patients, underlining the limitations of survey-based management assessment.

\section{DISCLOSURE}

Jeremie Calais is the recipient of a grant from the Fondation ARC pour la recherche sur le cancer (grant SAE20160604150). Wolfgang Fendler received a scholarship from the German Research Foundation (Deutsche Forschungsgemeinschaft, DFG, grant 807122). Matthias Eiber was supported by the SFB 824 (DFG Sonderforschungsbereich 824, Project B11) from the Deutsche Forschungsgemeinschaft, Bonn, Germany. Nicholas Nickols is a Prostate Cancer Foundation Young Investigator and a recipient of a VA Career Development Award (5IK2BX002520), a UCLA Prostate SPORE (4P50CA092131) Career Enhancement Award, a STOP Cancer Foundation Career Development Award, and a UCLA JCCC Seed Grant. Johannes Czernin is the recipient of a grant from the Prostate Cancer Foundation (2017 Challenge award; 17CHAL02) and the Johnson Comprehensive Cancer Center NIH-NCI Cancer Center Support Grant (P30 CA016042). Johannes Czernin is a founder and board member and holds equity in Sofie Biosciences and Trethera Therapeutics. Intellectual property has been patented by the University of California and has been licensed to Sofie Biosciences and Trethera Therapeutics. No other potential conflict of interest relevant to this article was reported. 


\section{REFERENCES}

1. Morigi JJ, Stricker PD, van Leeuwen PJ, et al. Prospective comparison of ${ }^{18} \mathrm{~F}$ fluoromethylcholine versus ${ }^{68} \mathrm{Ga}$-PSMA PET/CT in prostate cancer patients who have rising PSA after curative treatment and are being considered for targeted therapy. J Nucl Med. 2015;56:1185-1190.

2. Rauscher I, Maurer T, Beer AJ, et al. Value of ${ }^{68}$ Ga-PSMA HBED-CC PET for the assessment of lymph node metastases in prostate cancer patients with biochemical recurrence: comparison with histopathology after salvage lymphadenectomy. J Nucl Med. 2016;57:1713-1719.

3. Eiber M, Maurer T, Souvatzoglou M, et al. Evaluation of hybrid ${ }^{68}$ Ga-PSMA ligand $\mathrm{PET} / \mathrm{CT}$ in 248 patients with biochemical recurrence after radical prostatectomy. J Nucl Med. 2015;56:668-674.

4. Perera M, Papa N, Christidis D, et al. Sensitivity, specificity, and predictors of positive ${ }^{68} \mathrm{Ga}$-prostate-specific membrane antigen positron emission tomography in advanced prostate cancer: a systematic review and meta-analysis. Eur Urol. 2016;70:926-937.

5. Afshar-Oromieh A, Holland-Letz T, Giesel FL, et al. Diagnostic performance of ${ }^{68}$ Ga-PSMA-11 (HBED-CC) PET/CT in patients with recurrent prostate cancer: evaluation in 1007 patients. Eur J Nucl Med Mol Imaging. 2017;44:1258-1268.

6. Fendler WP, Calais J, Allen-Auerbach M, et al. ${ }^{68} \mathrm{Ga}$-PSMA-11 PET/CT interobserver agreement for prostate cancer assessments: an international multicenter prospective study. J Nucl Med. 2017;58:1617-1623.

7. Hillner BE, Siegel BA, Liu D, et al. Impact of positron emission tomography/ computed tomography and positron emission tomography (PET) alone on expected management of patients with cancer: initial results from the national oncologic PET registry. J Clin Oncol. 2008;26:2155-2161.

8. Hillner BE, Tosteson TD, Tosteson ANA, et al. Intended versus inferred management after PET for cancer restaging: analysis of Medicare claims linked to a coverage with evidence development registry. Med Care. 2013;51:361-367.

9. Calais J, Czernin J, Eiber M, et al. Most intended management changes after ${ }^{68}$ Ga-DOTATATE PET/CT are implemented. J Nucl Med. 2017;58:1793-1796.

10. Levine MN, Julian JA. Registries that show efficacy: good, but not good enough. $J$ Clin Oncol. 2008;26:5316-5319.

11. Larson SM. Practice-based evidence of the beneficial impact of positron emission tomography in clinical oncology. J Clin Oncol. 2008;26:2083-2084.

12. Albisinni S, Artigas C, Aoun F, et al. Clinical impact of ${ }^{68} \mathrm{Ga}$-prostate-specific membrane antigen (PSMA) positron emission tomography/computed tomography $(\mathrm{PET} / \mathrm{CT})$ in patients with prostate cancer with rising prostate-specific antigen after treatment with curative intent: preliminary analysis of a multidisciplinary approach. BJU Int. 2017;120:197-203.

13. Afaq A, Alahmed S, Chen $\mathrm{SH}$, et al. Impact of ${ }^{68} \mathrm{Ga}$-prostate-specific membrane antigen PET/CT on prostate cancer management. J Nucl Med. 2018;59:89-92.

14. Hope TA, Aggarwal R, Chee B, et al. Impact of Ga-68 PSMA-11 PET on management in patients with biochemically recurrent prostate cancer. $J$ Nucl Med. 2017;58:1956-1961.

15. Roach PJ, Francis R, Emmett L, et al. The impact of ${ }^{68} \mathrm{Ga}-\mathrm{PSMA}$ PET/CT on management intent in prostate cancer: results of an Australian prospective multicenter study. J Nucl Med. 2019;59:82-88.

16. Fendler WP, Eiber M, Beheshti M, et al. ${ }^{68} \mathrm{Ga}$-PSMA PET/CT: joint EANM and SNMMI procedure guideline for prostate cancer imaging: version 1.0. Eur J Nucl Med Mol Imaging. 2017;44:1014-1024.

17. Eder M, Schäfer M, Bauder-Wüst U, et al. ${ }^{68}$ Ga-complex lipophilicity and the targeting property of a urea-based PSMA inhibitor for PET imaging. Bioconjug Chem. 2012;23:688-697.

18. Schwarzenboeck SM, Rauscher I, Bluemel C, et al. PSMA ligands for PETimaging of prostate cancer. J Nucl Med. 2017;58:1545-1552.

19. Rauscher I, Maurer T, Fendler WP, Sommer WH, Schwaiger M, Eiber M. ${ }^{68} \mathrm{Ga}$-PSMA ligand PET/CT in patients with prostate cancer: how we review and report. Cancer Imaging. 2016;16:14.

20. Team RCR. A Language and Environment for Statistical Computing. Vienna, Austria: R Foundation for Statistical Computing; 2014.

21. Yang Y, Czernin J. Contribution of imaging to cancer care costs. J Nucl Med. 2011;52(suppl 2):86S-92S

22. Dinan MA, Curtis LH, Hammill BG, et al. Changes in the use and costs of diagnostic imaging among Medicare beneficiaries with cancer, 1999-2006. JAMA. 2010;303:1625-1631.

23. Mena E, Lindenberg ML, Shih JH, et al. Clinical impact of PSMA-based ${ }^{18} \mathrm{~F}-$ DCFBC PET/CT imaging in patients with biochemically recurrent prostate cancer after primary local therapy. Eur J Nucl Med Mol Imaging. 2018;45:4-11.

24. Seltzer MA, Yap CS, Silverman DH, et al. The impact of PET on the management of lung cancer: the referring physician's perspective. J Nucl Med. 2002;43:752756.

25. Cartwright A. Professionals as responders: variations in and effects of response rates to questionnaires, 1961-77. Br Med J. 1978;2:1419-1421.

26. Donaldson GW, Moinpour CM, Bush NE, et al. Physician participation in research surveys: a randomized study of inducements to return mailed research questionnaires. Eval Health Prof. 1999;22:427-441. 